\title{
Proposal for high-speed and high-fidelity electron-spin initialization in a negatively charged quantum dot coupled to a microcavity in a weak external magnetic field
}

\author{
Arka Majumdar, ${ }^{*}$ Ziliang Lin, Andrei Faraon, and Jelena Vučković \\ E. L. Ginzton Laboratory, Stanford University, Stanford, California 94305, USA
}

(Received 20 July 2009; published 2 August 2010)

\begin{abstract}
We describe a proposal for fast electron-spin initialization in a negatively charged quantum dot coupled to a microcavity without the need for a strong magnetic field. We employ two-photon excitation to access trion states that are spin forbidden by one-photon excitation. Our simulation shows a maximum initialization speed of $1.3 \mathrm{GHz}$ and maximum fidelity of $99.7 \%$ with realistic system parameters.
\end{abstract}

DOI: 10.1103/PhysRevA.82.022301

PACS number(s): 03.67.Lx, 78.67.Hc, 42.50.Pq, 73.21.La

Recent demonstrations of cavity quantum electrodynamics (QED) effects with semiconductor quantum dots (QDs) coupled to microcavities [1-5] show that these systems are robust and scalable platforms for quantum information science. The QD-cavity QED experiments performed so far treat each QD as a two-level quantum system consisting of a ground state and the single exciton excited state. However, multilevel quantum systems are useful for fast, complex, and high-fidelity quantum information processing [6]. Each of these systems should contain two stable ground states between which the transition is dipole forbidden and one or more excited states serve as a passage for population transfer between the ground states $(\Lambda$ system $)[7,8]$. Initialization, coherent manipulation, and readout of these $\Lambda$ systems are essential for quantum information processing, including controlled phase gate $[9,10]$, quantum repeaters and networks [11], and remote entanglement distribution [12]. Therefore, realizing a multilevel system in a QD coupled to a cavity is crucial for semiconductor cavity QED implementation of quantum computers.

One way to realize a $\Lambda$ system in a negatively charged QD is by employing the Zeeman-split electron-spin states as the ground states and a trion state as the excited state [6]. Recent experiments with QDs (not coupled to a cavity) have demonstrated spin initialization with magnetic field along the QD growth direction (Faraday geometry) [13] as well as with field perpendicular to the QD growth direction (Voigt geometry) $[7,14,15]$. Initialization in the Faraday geometry requires weak magnetic field and can achieve very high fidelity, but the initialization is slow because the process relies on random spin-flip Raman scattering. On the other hand, initialization in the Voigt geometry is fast, but a strong magnetic field is required to mix the spin states and split the resulting eigenstates for high fidelity. The high cost and large space required to achieve a strong magnetic field present a significant drawback of this initialization method.

In this paper, we propose a fast and high-fidelity spin initialization method for a negatively charged QD coupled to a microcavity. Our method also works for a positively charged QD [16], but we will focus on the negatively charged one as a specific example in this paper. The major advantage of our method is the absence of a strong magnetic field because there is no need to mix the electron or the hole spin states.

*arkam@stanford.edu
Instead, we employ a two-photon process to excite transitions that are otherwise spin forbidden by one-photon excitation. A doubly resonant cavity is used to enhance both the excitation and the spontaneous emission rates. We show that our method can achieve a maximum initialization speed of $1.3 \mathrm{GHz}$ and fidelity of $99.7 \%$ with realistic system parameters.

We consider a strongly confined QD and neglect the mixing between the light-hole and heavy-hole bands because of their large energy splitting. We model the QD potential as a finite well along its growth axis ( $z$ axis) and a two-dimensional oscillator perpendicular to its growth axis ( $x-y$ plane) $[17,18]$. We note that though the parabolic model is useful for analytical calculations, it excludes strain and piezoelectric effects [19]. A more accurate nonparabolic model of the confining potential can be obtained by full $\mathbf{k} \cdot \mathbf{p}$ simulations [20,21]. The effective mass for the electron (hole) is $m_{e}^{*}\left(m_{h}^{*}\right)$ and the oscillator frequency for the conduction (valence) band is $\omega_{e}\left(\omega_{h}\right)$. The harmonic-oscillator quantized levels are labeled as $s$ orbital, $p$ orbital, etc. [Fig. 1(a)]. Because the QD is much more confined along the $z$ axis than in the $x-y$ plane, the first several excited states have wave functions occupying different states in the $x-y$ plane but the same ground state along the $z$ axis [22].

Figure 1(b) shows the relevant QD energy levels for our initialization method. The ground levels are the electron spin up $\left|\uparrow_{s}\right\rangle$ and spin down $\left|\downarrow_{s}\right\rangle$ in the $s$ orbital. The application of a weak magnetic field of $0.2 \mathrm{~T}$ reduces the hyperfine-induced spin-flip rate $\gamma_{12}$ but does not mix the two spin states [23,24]. The first excited state is the trion state $\left|\uparrow_{s} \downarrow_{s} \uparrow_{s}\right\rangle$ with the electrons in the conduction-band $s$ orbital and the hole in the valence-band $s$ orbital. The second excited states are trion states generated by two-photon excitation $\left|\uparrow_{s} \uparrow_{p} \Uparrow_{s}\right\rangle$ and $\left|\downarrow_{s} \uparrow_{p} \uparrow_{s}\right\rangle$ in which one of the electrons is in the conductionband $p$ orbital.

The spin initialization to state $|\uparrow s\rangle$ is performed as follows: Two-photon excitation with $\sigma^{+}$circularly polarized light transfers population from $\left|\uparrow_{s}\right\rangle$ to $\left|\uparrow_{s} \uparrow_{p} \uparrow_{s}\right\rangle$ and from $\left|\downarrow_{s}\right\rangle$ to $\left|\downarrow_{s} \uparrow_{p} \Uparrow_{s}\right\rangle$ with a rate $\Omega$. The electron in the conduction-band $p$ orbital then relaxes to the conduction-band $s$ orbital by a phonon-assisted process with a typical time scale of 10-30 ps [25]. It should be noted that during this relaxation process, the hole spin can also be flipped. However, the hole spin-flip time is much longer $(\sim 1 \mathrm{~ms})$ [16] compared to the intersubband relaxation time. Hence we neglected the hole spin flip in our analysis. Due to the Pauli exclusion principle, the resulting state after intersubband relaxation is $\left|\uparrow_{s} \downarrow_{s} \uparrow_{s}\right\rangle$. The population 
(a)

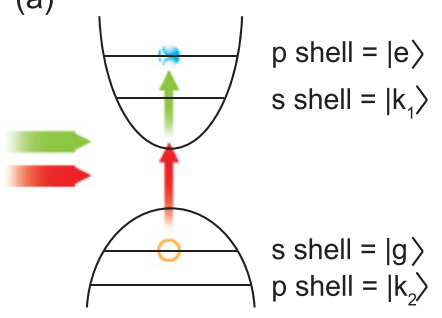

(b)

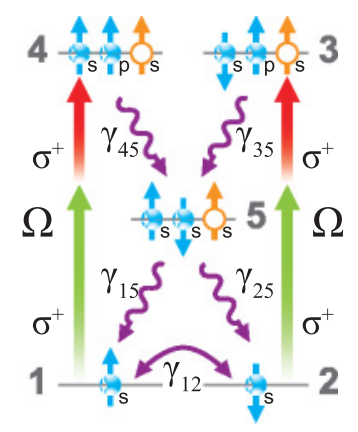

FIG. 1. (Color online) (a) Quantized energy levels of a neutral QD. The potential in the $x-y$ plane (perpendicular to the QD growth axis) is modeled as a two-dimensional harmonic oscillator. Upon two-photon absorption, an electron is excited from the valence-band $s$ orbital $(|g\rangle)$ to the conduction-band $p$ orbital ( $|e\rangle)$. (b) Energy-level diagram of relevant electron and trion states in a negatively charged QD. Electrons (holes) are shown in solid (opened) circles. Subscripts indicate orbital labels. The ground levels are $\left|\uparrow_{s}\right\rangle \equiv|1\rangle$ and $\left|\downarrow_{s}\right\rangle \equiv$ $|2\rangle$. The first excited state is the trion state $\left|\uparrow_{s} \downarrow_{s} \uparrow_{s}\right\rangle \equiv|5\rangle$. The second excited states are trion states excited by two-photon processes $\left|\uparrow_{s} \uparrow_{p} \uparrow_{s}\right\rangle \equiv|4\rangle$ and $\left|\downarrow_{s} \uparrow_{p} \Uparrow_{s}\right\rangle \equiv|3\rangle$. The straight arrows indicate two-photon excitation, the curly arrows indicate various decays of excited states, and the curved arrow indicates spin flip.

in this state will preferentially decay to the state $\left|\uparrow_{s}\right\rangle$ with rate $\Gamma \equiv \gamma_{15}$ because the decay to state $\left|\downarrow_{s}\right\rangle$ is spin forbidden. Eventually, the population accumulates in the state $|\uparrow s\rangle$. Two-photon excitation cannot directly create an electron and a hole in the conduction- and valence-band $s$ orbitals as these two orbitals have different parity. For this reason, we use the $p$ orbital for intermediate states. However, one can avoid excitation to $p$ orbitals by applying a lateral electric field across the QD and thus changing the parity of the wave functions of the $s$ orbitals [26].

In order to achieve fast initialization, a doubly resonant cavity is needed to enhance the QD spontaneous decay rate $\Gamma$ and the two-photon excitation rate $\Omega$. We consider InAs/GaAs QDs with an $s-s$ transition wavelength of $930 \mathrm{~nm}$. Since the $p$ orbital is approximately $50 \mathrm{meV}$ above the $s$ orbital in the conduction band [22], this implies that the $s-p$ transition wavelength is approximately $900 \mathrm{~nm}$. Therefore, we need a doubly resonant cavity at $930 \mathrm{~nm}$ and $1800 \mathrm{~nm}$ to enhance the spontaneous decay rate and the two-photon excitation rate, respectively. Both resonant modes should be polarization degenerate because all the selection rules described in this paper require circularly polarized light. The electric field for the two polarization degenerate modes should also have significant spatial overlap around the QD so that the QD can be excited via a circularly polarized light. Designs of high- $Q$ photonic crystal cavities, whose doubly degenerate modes have a strong spatial overlap, have already been investigated [27].

We follow Ref. [26] to estimate the two-photon excitation rate of a QD inside a microcavity. When both photons are of the same frequency, the cavity-enhanced two-photon absorption rate is $\Gamma_{\omega_{c}}^{\mathrm{TPA}}=2 \pi|\Omega|^{2} \delta\left(\omega_{d}-2 \omega\right)$, where the effective Rabi rate $\Omega$ is

$$
\Omega=\frac{\eta P Q \phi}{4 \hbar^{2} \omega n^{2} \varepsilon_{0} V} \sum_{k} \frac{\left|\mathbf{d}_{g k}\right|\left|\mathbf{d}_{k e}\right| \psi_{g k} \psi_{k e}}{\Delta_{g k}},
$$

where $\eta, P, Q, n$, and $V$ are the light-coupling efficiency into the cavity, excitation power (measured outside the cavity), cavity quality factor, refractive index of material that the cavity is composed of, and cavity mode volume, respectively. $\omega_{c}$, $\omega_{d}$, and $\omega$ are the cavity resonance frequency, the transition frequency between two QD levels coupled by the two-photon process, and the laser frequency, respectively. $\mathbf{d}_{g k}\left(\mathbf{d}_{k e}\right)$ is the dipole moment between the ground (excited) state and the intermediate state $k . \Delta_{g k}=\omega_{k}-\omega_{g}-\omega$ is the laser detuning from the transition between the QD ground state and the intermediate state. The delta function $\delta\left(\omega_{d}-2 \omega\right)$ represents energy conservation. The factor $\phi$ describes the spectral mismatch between the laser and the cavity, $\phi(\omega)=$ $\frac{\omega / \omega_{c}}{1+4 Q^{2}\left(\omega / \omega_{c}-1\right)^{2}}$. The factor $\psi_{g k}\left(\psi_{k e}\right)$ characterizes the reduction in Rabi rate due to position mismatch between the QD and the electric-field maximum within the cavity, $\psi_{g k}=$ $|\mathbf{E}(\mathbf{r})|\left|\mathbf{E}\left(\mathbf{r}_{M}\right)\right|\left(\mathbf{d}_{g k} \cdot \hat{\mathbf{e}}\left|\mathbf{d}_{g k}\right|\right)$, where $\mathbf{E}(\mathbf{r}), \mathbf{E}\left(\mathbf{r}_{M}\right)$, and $\hat{\mathbf{e}}$ are the electric field at $\mathbf{r}$, electric field maximum, and electric field polarization at the QD location, respectively.

For the transition from the valence-band $s$ orbital to the conduction-band $p$ orbital, the dominant intermediate states are the conduction-band $s$ orbital $\left|k_{1}\right\rangle=\left|s_{c}\right\rangle$ and the valenceband $p$ orbital $\left|k_{2}\right\rangle=\left|p_{v}\right\rangle$ [Fig. 1(a)]. In our analysis, we assume $\left|\mathbf{d}_{g k_{1}}\right|=\left|\mathbf{d}_{e k_{2}}\right|=e\left|\mathbf{r}_{c v}\right|\left(\left|\mathbf{r}_{c v}\right|=0.6 \mathrm{~nm}\right.$ [28]), which is the transition moment between the valence band and the conduction band integrated over a unit cell. We also calculate $\left|\mathbf{d}_{e k_{1}}\right|=e l_{e}$ and $\left|\mathbf{d}_{g k_{2}}\right|=e l_{h}$, where $e$ is the electronic charge and $l_{e}$ and $\left(l_{h}\right)$ is the oscillating length of the electron (hole) with $l_{e}=\sqrt{\hbar /\left(2 m_{e}^{*} \omega_{e}\right)}\left[l_{h}=\sqrt{\hbar /\left(2 m_{h}^{*} \omega_{h}\right)}\right]$. With $\eta=2 \%$, $Q=5000, V=(\lambda / n)^{3}, \phi=1, \psi=1$, and $P=50 \mu \mathrm{W}$, we calculate $\Omega / 2 \pi=4.5 \mathrm{GHz}$. We note that we use a very conservative number of $2 \%$ for in-coupling efficiency into the cavity, as in our previous experiments on controlling cavity reflectivity with a single QD [1], but significantly higher coupling efficiency exceeding $80 \%$ is possible with fiber tapers or cavity-waveguide couplers $[29,30]$. Therefore, much higher $\Omega$ is possible with a smaller excitation power.

The fidelity and speed of our spin initialization method are analyzed using a method based on the master equation [31]. Here we analyze the case when $\sigma^{+}$light is applied. The time evolution of the density matrix $\rho$ of the $\mathrm{QD}$ is given by

$$
\frac{d \rho}{d t}=-\frac{i}{\hbar}[\mathcal{H}, \rho]+\sum_{j} \mathcal{L}_{j}(\rho)
$$

with the Hamiltonian

$$
\mathcal{H}=\hbar \Omega\left(\left|\uparrow_{s} \uparrow_{p} \Uparrow_{s}\right\rangle\left\langle\uparrow_{s}|+| \downarrow_{s} \uparrow_{p} \Uparrow_{s}\right\rangle\left\langle\downarrow_{s}\right|\right)+\text { H.c. }
$$

The Zeeman splitting between the states $\left|\uparrow_{s}\right\rangle$ and $\left|\downarrow_{s}\right\rangle$ is approximately $5.4 \mu \mathrm{eV}$ for an electronic $g$ factor of 0.46 [15] and a magnetic field of $0.2 \mathrm{~T}$. This splitting is much smaller than $\hbar \Omega$ and $\hbar \Gamma$, therefore we neglect it in our analysis. $\mathcal{L}_{j}(\rho) \equiv D_{j} \rho D_{j}^{\dagger}-\frac{1}{2} D_{j}^{\dagger} D_{j} \rho-\frac{1}{2} \rho D_{j}^{\dagger} D_{j}$, where $D$ 's are the collapse operators:

$$
\begin{gathered}
D_{1}=\sqrt{\Gamma}\left|\uparrow_{s}\right\rangle\left\langle\uparrow_{s} \downarrow_{s} \uparrow_{s}\right|, \\
D_{2}=\sqrt{\gamma_{12}}\left|\downarrow_{s}\right\rangle\left\langle\uparrow_{s}\right|, \\
D_{3}=\sqrt{\gamma_{25}}\left|\downarrow_{s}\right\rangle\left\langle\uparrow_{s} \downarrow_{s} \Uparrow_{s}\right|,
\end{gathered}
$$




$$
\begin{aligned}
& D_{4}=\sqrt{\gamma_{35}}\left|\uparrow_{s} \downarrow_{s} \uparrow_{s}\right\rangle\left\langle\downarrow_{s} \uparrow_{p} \uparrow_{s}\right|, \\
& D_{5}=\sqrt{\gamma_{45}}\left|\uparrow_{s} \downarrow_{s} \uparrow_{s}\right\rangle\left\langle\uparrow_{s} \uparrow_{p} \uparrow_{s}\right| .
\end{aligned}
$$

Numerical values of the rates used for the simulation are $\gamma_{25} / 2 \pi=100 \mathrm{kHz}[13], \gamma_{12} / 2 \pi=10 \mathrm{kHz}$, and $\gamma_{35} / 2 \pi=$ $\gamma_{45} / 2 \pi=8 \mathrm{GHz}$, corresponding to a relaxation time of $20 \mathrm{ps}$ [Fig. 1(b)]. The QD spontaneous emission rate $\Gamma$ is Purcell enhanced and its numerical value ranges from 5 to $20 \mathrm{GHz}$, corresponding to a Purcell factor ranging from 50 to 200.

The fidelity and initialization speed have a strong dependence on $\Omega$ and $\Gamma$. Experimentally, $\Omega$ is tuned by changing the excitation light intensity and by employing cavity resonance at two-photon excitation frequency; $\Gamma$ is tuned by placing the QD in nanocavities of various quality factors. To find the fidelity, the steady-state density matrix is solved. The fidelity is defined as the population in state $\left|\uparrow_{s}\right\rangle$, namely $\rho_{11}$. To find the initialization speed, the density operator is evolved in time from the initial condition of $\rho_{11}(t=0)=\rho_{22}(t=0)=1 / 2$, where $\rho_{22}$ is the population in the state $\left|\downarrow_{s}\right\rangle$. The initialization time is defined as the time required for $\rho_{11}(t)$ to reach the value of $1-1 / e$.

Figure 2(a) shows the initialization fidelity as a function of the two-photon effective Rabi rate $\Omega$ and the one-photon spontaneous emission rate $\Gamma$. For fixed $\Gamma$, increasing $\Omega$ depletes population from state $\left|\downarrow_{s}\right\rangle$ more efficiently and therefore leads to increasing population in state $\left|\uparrow_{s}\right\rangle$ and thus higher fidelity. However, while depleting population from state $\left|\downarrow_{s}\right\rangle$, two-photon excitation also depletes population from state

(a)

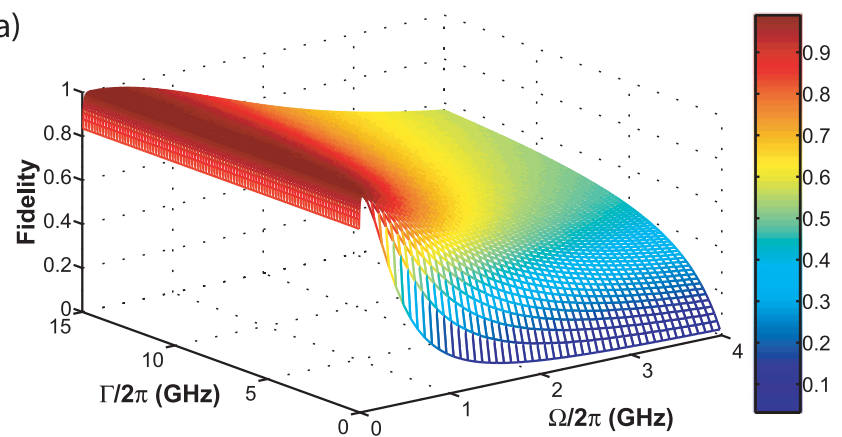

(b)

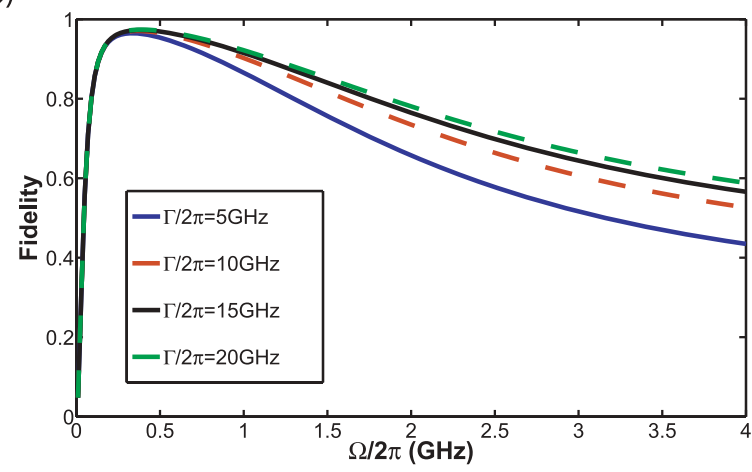

FIG. 2. (Color online) (a) Fidelity of electron-spin initialization as a function of the two-photon excitation rate $\Omega / 2 \pi$ and the spontaneous emission rate $\Gamma / 2 \pi$. (b) Slices through plot (a) for specific $\Gamma / 2 \pi$ values (the legend shows the curves from bottom to top), indicating a maximum attainable fidelity of $99.7 \%$. $|\uparrow s\rangle$ [Fig. 1(b)]. Therefore, there are two competing processes: one depletes the population in $\left|\uparrow_{s}\right\rangle$ with a rate of $\Omega$ and the other populates $\left|\uparrow_{s}\right\rangle$ with a rate of $\Gamma$. Hence, increasing $\Omega$ indefinitely for a fixed $\Gamma$ depletes $\left|\uparrow_{s}\right\rangle$ and ultimately decreases the fidelity of initialization [Figs. 2(a) and 2(b)]. Such a reduction in fidelity is more prominent for lower $\Gamma$. On the other hand, for fixed $\Omega$, increasing $\Gamma$ allows faster population transfer from state $\left|\uparrow_{s} \downarrow_{s} \uparrow_{s}\right\rangle$ to state $\left|\uparrow_{s}\right\rangle$, leading to higher fidelity. The maximum fidelity obtained is $99.7 \%$. This fidelity is comparable to the fidelity of initialization achieved in the Faraday geometry $(\geqslant 99.8 \%)[13]$ at a magnetic field of $0.2 \mathrm{~T}$ and in the Voigt geometry $(\approx 99.7 \%)$ at a magnetic field of $1 \mathrm{~T}$ [15]. It should be noted that the spin-flip rate $\gamma_{12}$ also limits the fidelity. For a spin-flip rate increased from $\gamma_{12} / 2 \pi=10 \mathrm{kHz}$ (as in Fig. 2) to $100 \mathrm{kHz}$ and $1 \mathrm{MHz}$, the maximum fidelity decreases to $99 \%$, and $98 \%$, respectively.

Figure 3(a) shows the time needed to perform spin initialization as a function of $\Omega$ and $\Gamma$. As shown in Fig. 2(b), an increase in $\Omega / \Gamma$ results in a decrease in the fidelity. In fact, for $\Omega / \Gamma>0.2$, the fidelity never reaches the value of $1-1 / e$ and therefore initialization time is arbitrarily long. For this reason, we study only the range $\Omega / \Gamma<0.2$ in Fig. 3(b). It should be emphasized that the value 0.2 is obtained for the parameters used in the simulation, and a different value will be obtained for a different set of parameters. As shown in Fig. 3(b), when $\Omega / \Gamma<0.2$, the initialization speed increases with increasing $\Omega / \Gamma$. Although increasing $\Gamma$ in general increases the speed, this speed increase is not significant when $\Gamma \gg \gamma_{35}=\gamma_{45}$ because the phonon-assisted relaxation rates from $\left|\uparrow_{s} \uparrow_{p} \Uparrow_{s}\right\rangle$ and $\left|\downarrow_{s} \uparrow_{p} \Uparrow_{s}\right\rangle$ to $\left|\uparrow_{s} \downarrow_{s} \uparrow_{s}\right\rangle$ become the limiting factor. The minimum initialization time calculated is $120 \mathrm{ps}$, giving the

(a)

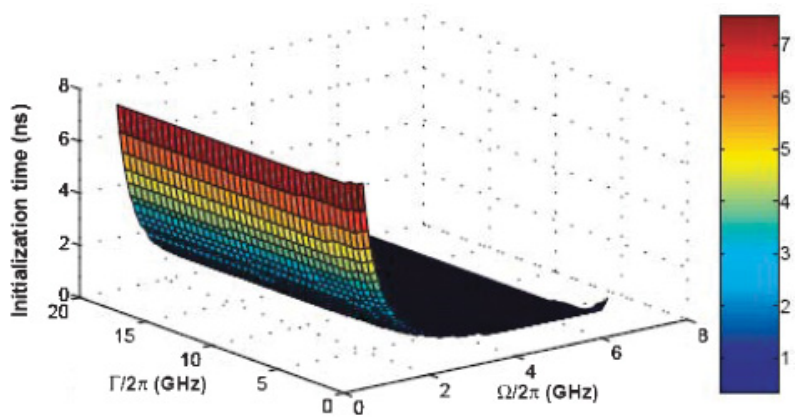

(b)

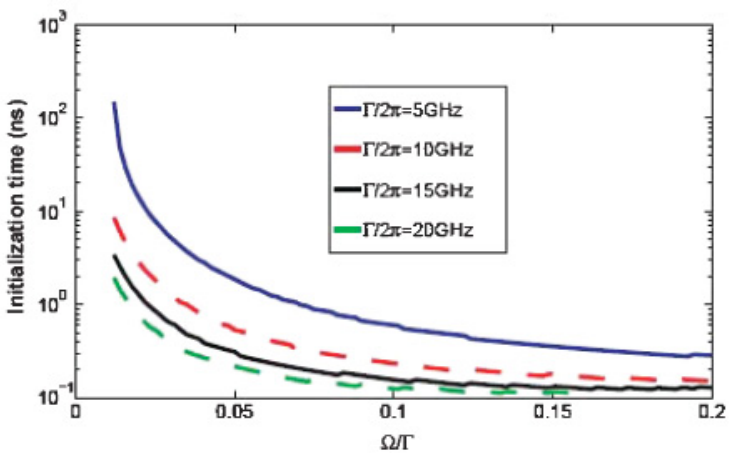

FIG. 3. (Color online) (a) Time needed to perform electron-spin initialization as a function of $\Omega$ and $\Gamma$. (b) Initialization time as a function of the ratio of $\Omega$ and $\Gamma$ for different values of $\Gamma$ (the legend shows curves from top to bottom). 
maximum speed of $1.3 \mathrm{GHz}$. This speed of initialization is much higher than the maximum speed obtained in the Faraday geometry (100 kHz) [13] and the Voigt geometry (144 MHz) [15]. It should be noted that the speed in Voigt geometry can potentially be enhanced by using a cavity, but that is not true for Faraday geometry. Unlike fidelity, a change in the spin-flip rate $\gamma_{12}$ does not affect the speed of initialization, as $\gamma_{12} \ll \Omega, \Gamma$.

It is important to note the trade off between high speed and high fidelity. Figure 3 shows that a high two-photon excitation rate $\Omega$ is needed to achieve high speed, but subsequently the fidelity decreases at high $\Omega$ (Fig. 2). Therefore, the speed of initialization corresponding to the maximum fidelity of $99.7 \%$ is lower $(\approx 50 \mathrm{MHz})$. Similarly, at the highest initialization rate, the fidelity of only $80 \%$ can be achieved. However, we can still achieve moderate fidelity with moderate speed: for example with $\Omega / 2 \pi=0.5 \mathrm{GHz}$ and $\Gamma / 2 \pi=$ $10 \mathrm{GHz}$ we can achieve a fidelity of $97.3 \%$ and a speed of $320 \mathrm{MHz}$.

In conclusion, we have described a method for initializing electron spin in a negatively charged QD using cavity-enhanced two-photon excitation. Under the assumption of a parabolic confining potential and noninteracting electrons and holes, the highest speed of $1.3 \mathrm{GHz}$ and the maximum fidelity of $99.7 \%$ can be achieved with a weak magnetic field of approximately $0.2 \mathrm{~T}$ and realistic system parameters. Although it is not possible to achieve both the maximum speed and fidelity simultaneously, with $\Omega / 2 \pi=0.5 \mathrm{GHz}$ and $\Gamma / 2 \pi=10 \mathrm{GHz}$, one can achieve a fidelity of $97.3 \%$ together with a speed of $320 \mathrm{MHz}$. The use of a weak magnetic field reduces the cost and the space requirements for a quantum network. A magnetic field of $0.2 \mathrm{~T}$ can easily be achieved with a small electromagnet as opposed to using a large superconducting magnet needed for a strong magnetic field in other spin initialization techniques.

The authors gratefully acknowledge financial support provided by the Army Research Office, National Science Foundation, and Office of Naval Research. A.M. was supported by Texas Instruments. Z. L. was supported by the National Science Foundation.
[1] D. Englund, A. Faraon, I. Fushman, N. Stoltz, P. Petroff, and J. Vučković, Nature (London) 450, 857 (2007).

[2] K. Hennessy, A. Badolato, M. Winger, D. Gerace, M. Atature, S. Gulde, S. Falt, E. L. Hu, and A. Imamoglu, Nature (London) 445, 896 (2007).

[3] K. Srinivasan and O. Painter, Nature (London) 450, 862 (2007).

[4] D. Press, S. Götzinger, S. Reitzenstein, C. Hofmann, A. Löffler, M. Kamp, A. Forchel, and Y. Yamamoto, Phys. Rev. Lett. 98, 117402 (2007).

[5] T. Yoshie, A. Scherer, J. Hendrickson, G. Khitrova, H. M. Gibbs, G. Rupper, C. Ell, O. B. Shchekin, and D. G. Deppe, Nature (London) 432, 200 (2004).

[6] A. Imamoglu, D. D. Awschalom, G. Burkard, D. P. DiVincenzo, D. Loss, M. Sherwin, and A. Small, Phys. Rev. Lett. 83, 4204 (1999).

[7] D. Press, T. D. Ladd, B. Zhang, and Y. Yamamoto, Nature (London) 456, 218 (2008).

[8] J. A. Gupta, R. Knobel, N. Samarth, and D. D. Awschalom, Science 292, 2458 (2001).

[9] L.-M. Duan and H. J. Kimble, Phys. Rev. Lett. 92, 127902 (2004).

[10] I. Fushman, D. Englund, A. Faraon, N. Stoltz, P. Petroff, and J. Vučković, Science 320, 769 (2008).

[11] J. I. Cirac, P. Zoller, H. J. Kimble, and H. Mabuchi, Phys. Rev. Lett. 78, 3221 (1997).

[12] C. W. Chou, H. de Riedmatten, D. Felinto, S. V. Polyakov, S. J. van Enk, and H. J. Kimble, Nature (London) 438, 828 (2005).

[13] M. Atature, J. Dreiser, A. Badolato, A. Hogele, K. Karrai, and A. Imamoglu, Science 312, 551 (2006).

[14] X. Xu, Y. Wu, B. Sun, Q. Huang, J. Cheng, D. G. Steel, A. S. Bracker, D. Gammon, C. Emary, and L. J. Sham, Phys. Rev. Lett. 99, 097401 (2007).

[15] C. Emary, X. Xu, D. G. Steel, S. Saikin, and L. J. Sham, Phys. Rev. Lett. 98, 047401 (2007).
[16] B. D. Gerardot, D. Brunner, P. A. Dalgarno, P. Ohberg, S. Seidl, M. Kroner, K. Karrai, N. G. Stoltz, P. M. Petroff, and R. J. Warburton, Nature (London) 451, 441 (2008).

[17] M. Korkusinski, M. E. Reimer, R. L. Williams, and P. Hawrylak, Phys. Rev. B 79, 035309 (2009).

[18] M. E. Reimer, M. Korkusiński, D. Dalacu, J. Lefebvre, J. Lapointe, P. J. Poole, G. C. Aers, W. R. McKinnon, P. Hawrylak, and R. L. Williams, Phys. Rev. B 78, 195301 (2008).

[19] M. Grundmann, O. Stier, and D. Bimberg, Phys. Rev. B 52, 11969 (1995).

[20] C. Pryor, Phys. Rev. B 57, 7190 (1998).

[21] O. Stier, M. Grundmann, and D. Bimberg, Phys. Rev. B 59, 5688 (1999).

[22] P. Michler, Single Quantum Dots: Fundamentals, Applications and New Concepts (Topics in Applied Physics) (Springer, 2003).

[23] J. M. Elzerman, R. Hanson, L. H. W. van Beveren, B. Witkamp, L. M. K. Vandersypen, and L. P. Kouwenhoven, Nature (London) 430, 431 (2004).

[24] M. Kroutvar et al., Nature (London) 432, 81 (2004).

[25] S. Sauvage, P. Boucaud, R. P. S. M. Lobo, F. Bras, G. Fishman, R. Prazeres, F. Glotin, J. M. Ortega, and J.-M. Gérard, Phys. Rev. Lett. 88, 177402 (2002).

[26] Z. Lin and J. Vučković, Phys. Rev. B 81, 035301 (2010).

[27] K. Hennessy, C. Hogerle, E. Hu, A. Badolato, and A. Imamoglu, Appl. Phys. Lett. 89, 041118 (2006).

[28] P. G. Eliseev, H. Li, A. Stintz, G. T. Liu, T. C. Newell, K. J. Malloy, and L. F. Lester, Appl. Phys. Lett. 77, 262 (2000).

[29] A. Faraon, D. Englund, I. Fushman, J. Vučković, and E. Waks, Appl. Phys. Lett. 90, 073102 (2007).

[30] I.-K. Hwang, S.-K. Kim, J.-K. Yang, S.-H. Kim, S. H. Lee, and Y.-H. Lee, Appl. Phys. Lett. 87, 131107 (2005).

[31] C. W. Gardiner and P. Zoller, Quantum Noise: A Handbook of Markovian and Non-Markovian Quantum Stochastic Methods with Applications to Quantum Optics (Springer Series in Synergetics, 2005). 